\title{
Relative Lagrangian Formulation of Finite Thermoelasticity
}

\author{
I-Shih Liu and Marcello G. Teixeira \\ Instituto de Matemática, Universidade Federal \\ do Rio de Janeiro, Brasil
}

\begin{abstract}
Besides the Lagrangian and the Eulerian descriptions, the motion of a body can also be expressed relative to the present configuration of the body, known as the relative motion description. It is interesting to consider such a relative motion description in general to formulate the basic system of field equations for solid bodies. In doing so, when the time increment from the present state is small enough, the nonlinear constitutive equations can be linearized relative to the present state so that the resulting system becomes linear. This will be done for thermoelastic materials with a brief comment on the exploitation of entropy principle in general.

Relative Lagrangian formulation is based on the well-known "small-on-large" idea, and can be implemented for solving problems with large deformation in successive incremental manner. Some applications of such a formulation in numerical simulations are briefly reviewed.
\end{abstract}

Keywords: Relative Lagrangian formulation, thermoelastic solid, small on large, successive linear approximation, boundary value problem.

\section{Introduction}

In modern continuum thermodynamics, to deduce thermodynamic restrictions on constitutive functions two essential approaches are widely employed, i.e., ColemanNoll procedure and Müller-Liu procedure. The two procedures are based on different assumptions, for which the later is more general than the former, so that the resulting restrictions may not be identical in general. Nevertheless, it turns out that they do give the same results for the most usual materials in practical application, such as elastic and viscous heat-conducting fluids, isotropic thermoelastic and thermoviscoelastic solids. However, it is no so for thermoelastic materials in general, for which the employment of Coleman-Noll and Müller-Liu procedures in analyzing the entropy principle will be briefly discussed.

The governing equations for a material body are usually formulated in Lagrangian or Eulerian domains, and for a solid body, the Lagrangian formulation relative to some reference state is more convenient. Theoretically, any state can be chosen as 
a reference state for a Lagrangian description. Indeed, with the real motion of the body at the present time as the reference state is usually known as the relative motion description (see for example, [22, 7]). It is interest to consider a formulation with relative motion description for solid bodies in general. We shall refer this as relative Lagrangian formulation in contrast to (total) Lagrangian formulation with respect to a fixed reference state.

By doing so, when the time increment from the present state is small enough, it is then possible to linearize the constitutive equation relative to the present state so that the governing equation of the problem becomes linear partial differential equation for small deformation. This idea is similar to the well-known problem of small deformation superposed on finite deformation (small-on-large) in the literature [5]. To be more specific, thermoelastic material bodies will be considered in details. It constitutes a straight generalization of the relative Lagrangian formulation of elastic bodies in [11].

We can then propose a linear algorithm for large deformation based on relative Lagrangian formulation, by building up successive small incremental deformation problem at every time step in the deformation process. Roughly speaking, at each time step, the constitutive function is calculated at the present state of deformation which will be regarded as the reference configuration for the next state, and assuming the deformation to the next state is small, the constitutive function can be linearized. In this manner, it becomes a linear problem at each time step from one state to the next state successively with small deformations. This will be referred to as the successive linear approximation. The application of the successive linear approximation for some material models will be briefly reviewed.

\section{Thermodynamics of elastic materials}

Let $\kappa_{0}$ be a fixed reference configuration of the body $\mathcal{B}$. For $X \in \kappa_{0}(\mathcal{B})$ and time $t \in$ $\mathbb{R}$, we consider the basic fields of a material body, the density $\rho(X, t)$, the motion $\boldsymbol{x}=\chi(X, t)$ and the temperature $\theta(X, t)$.

\subsection{Governing equations}

The equations of balance of mass, linear momentum and the internal energy are given by

$$
\begin{aligned}
& \dot{\rho}+\rho \operatorname{div} \dot{\boldsymbol{x}}=0, \\
& \rho \ddot{\boldsymbol{x}}-\operatorname{div} T=\rho \boldsymbol{b}, \\
& \rho \dot{\varepsilon}+\operatorname{div} \boldsymbol{q}-T \cdot \operatorname{grad} \dot{\boldsymbol{x}}=\rho r,
\end{aligned}
$$

where $\boldsymbol{b}$ is the external body force and $r$ is the external energy supply. The equations are stated in Eulerian description, in which the divergence and the gradient operators are relative to the deformed configuration at time $t$.

For thermoelastic bodies, the constitutive equations for the Cauchy stress tensor, 
the heat flux vector and the internal energy density can be expressed respectively as

$$
T=T(F, \theta, \boldsymbol{g}), \quad \boldsymbol{q}=\boldsymbol{q}(F, \theta, \boldsymbol{g}), \quad \varepsilon=\varepsilon(F, \theta, \boldsymbol{g}),
$$

where $F=\nabla_{X} \boldsymbol{x}$ and $\boldsymbol{g}=\nabla_{X} \theta$ are the deformation gradient and the temperature gradient with respect to the fixed reference configuration $\kappa_{0}$.

The governing equations for the basic fields consist of the balance laws (1) together with the constitutive equations (2) for a particular class of material bodies. Any solution of the governing equations is called a thermodynamic process.

\subsection{Entropy principle}

In the framework of continuum mechanics, there are some universal restrictions for a mathematical formulation of a physical theory of material bodies, among them, the entropy principle, which states that any thermodynamic process must satisfy the entropy inequality,

$$
\rho \dot{\eta}+\operatorname{div} \Phi-\rho s \leq 0,
$$

where $\eta$ is the entropy density, $\Phi$ is the entropy flux and $s$ is the entropy supply.

Motivated by the classical thermostatics, it is often assume that

$$
\Phi=\frac{1}{\theta} \boldsymbol{q}, \quad s=\frac{1}{\theta} r,
$$

and the entropy inequality (3) becomes

$$
\rho \dot{\eta}+\operatorname{div}\left(\frac{\boldsymbol{q}}{\theta}\right)-\rho\left(\frac{r}{\theta}\right) \leq 0,
$$

known as Clausius-Duhem inequality.

Since the balance laws are universal for any material bodies, the entropy principle requirement effectively places severe restrictions on the constitutive equations of a proposed material model so that any thermodynamic process would satisfy the entropy inequality accordingly. Consequently, the exploitation of such restrictions is the essential objective of formulating a thermodynamic theory of any material model.

Such general thermodynamic restrictions for thermoelastic material bodies can be stated in the following proposition ([3, 6, 7, 9]):

Proposition. For thermoelastic material bodies, the Cauchy stress, the internal energy and the entropy are related to the free energy function $\psi=\psi(F, \theta)$ by

$$
T=\rho \frac{\partial \psi}{\partial F} F^{T}, \quad \varepsilon=\psi-\theta \frac{\partial \psi}{\partial \theta}, \quad \eta=-\frac{\partial \psi}{\partial \theta},
$$

which characterizes the thermoelastic bodies as hyperelastic. Moreover, the heat flux $\boldsymbol{q}=\boldsymbol{q}(F, \theta, \boldsymbol{g})$ and the entropy flux satisfy

$$
\boldsymbol{q} \cdot \boldsymbol{g} \leq 0, \quad \Phi=\frac{1}{\theta} \boldsymbol{q}
$$


Therefore, as a consequence of entropy principle requirement, the general constitutive equations of the stress, the internal energy and the entropy for thermoelastic bodies are independent of the temperature gradient,

$$
T=T(F, \theta), \quad \varepsilon=\varepsilon(F, \theta), \quad \eta=\eta(F, \theta) .
$$

Moreover, from (6), they satisfy the Gibb's relation,

$$
d \eta=\frac{1}{\theta}\left(d \varepsilon-\frac{1}{\rho} T F^{-T} \cdot d F\right)
$$

From this relation, we can write

$$
\dot{\varepsilon}=\theta \dot{\eta}+\frac{1}{\rho} T \cdot \dot{F} F^{-1},
$$

and since $\operatorname{grad} \dot{\boldsymbol{x}}=\dot{F} F^{-1}$, the energy equation (1) ${ }_{3}$ can be rewritten as

$$
\rho \theta \dot{\eta}+\operatorname{div} \boldsymbol{q}=\rho r
$$

which is a convenient form of the energy equation for thermoelastic bodies.

\subsection{Remarks on the exploitation of the entropy principle}

Exploitation of entropy principle based on the Clausius-Duhem inequality (5) has been widely adopted in the development of modern continuum thermodynamics following the Coleman-Noll procedure $([3,6,7])$. In particular, the general restrictions for elastic materials are stated in the above Proposition.

However, the main assumptions (4) for Clausius-Duhem inequality might seem to be plausible in most classical theories of continuum mechanics, are not particularly well motivated for materials in general as pointed out by Müller [16]. To deal with the exploitation based on the general entropy inequality (3), the Müller-Liu procedure $([7,9,10])$ by the use of Lagrange multipliers [8] can be employed.

For isotropic thermoelastic materials [9], both the Müller-Liu procedure and the Coleman-Noll procedure do lead to the same Proposition above. However, for thermoelastic materials in general, it has been noted that such results cannot be proved mathematically without some additional assumptions.

Indeed, for transversely isotropic thermoelastic materials [10], it has been proved that the classical flux relation does not hold, i.e., the entropy flux may not be proportional to the heat flux and the relation (7) does not hold. Nevertheless, it has also been proved that the relations (6) remain valid. In other words, the transverly isotropic thermoelastic materials are still being hyperelastic, irrespective of whether the classical entropy flux relation is valid or not. To what extent that a thermoelastic material body is hyperelastic based on the general entropy inequality (3) remains an open question. 


\subsection{Balance equations in Lagrangian description}

For solid bodies, boundary value problems are usually formulated relative to some reference configuration. Although in practice, the reference configuration is chosen as the initial configuration, usually the preferred configuration for which the constitutive equations of the particular material class are given, it is by no means necessary. The Lagrangian formulation of boundary value problems can be formulated with respect to any reference configuration, in particular, even the present configuration of the body can be chosen if one so desires.

Let $\kappa$ be an arbitrary reference configuration of the body $\mathcal{B}$. The balance equations of mass, linear momentum and energy, with domain in $\kappa(\mathcal{B}) \times \mathbb{R}$, can be written as

$$
\begin{aligned}
& \rho_{\kappa}=\rho \operatorname{det} F_{\kappa}, \\
& \rho_{\kappa} \ddot{\boldsymbol{x}}-\operatorname{div}_{\kappa} T_{\kappa}=\rho_{\kappa} \boldsymbol{b}, \\
& \rho_{\kappa} \theta \dot{\eta}+\operatorname{div}_{\kappa} \boldsymbol{q}_{\kappa}=\rho_{\kappa} r,
\end{aligned}
$$

where $\operatorname{div}_{\kappa}$ denotes the divergence operator relative to the coordinate system of the configuration $\kappa$, and $F_{\kappa}$ is the deformation gradient with respect to the configuration $\kappa$. In these equations following definitions have been introduced,

$$
T_{\kappa}=\left(\operatorname{det} F_{\kappa}\right) T F_{\kappa}^{-T}, \quad \boldsymbol{q}_{\kappa}=\left(\operatorname{det} F_{\kappa}\right) F_{\kappa}^{-1} \boldsymbol{q} .
$$

In which $T_{\kappa}$ is called the (first) Piola-Kirchhoff stress tensor and $\boldsymbol{q}_{\kappa}$ is referred to as the material heat flux vector with respect to the reference configuration $\kappa$.

\section{The present state as reference configuration}

Since the choice of a reference configuration is arbitrary, we shall now consider a formulation that the present configuration is chosen as reference configuration. This is known in continuum mechanics as the relative motion description in contrast to the usual Eulerian and Lagrangian descriptions [22, 7].

Let $\kappa_{0}$ be the fixed reference configuration of the body $\mathcal{B}$ at time $t_{0}$, and $\kappa_{t}$ be its deformed configuration at the present time $t$, Let

$$
\boldsymbol{x}=\chi(X, t) \quad X \in \kappa_{0}(\mathcal{B}),
$$

and

$$
F(X, t)=\nabla_{X}(\chi(X, t))
$$

be the deformation and the deformation gradient from $\kappa_{0}$ to $\kappa_{t}$.

Now, at some time $\tau$, consider the deformed configuration $\kappa_{\tau}$,

$$
\boldsymbol{\xi}=\chi(X, \tau):=\chi_{t}(\boldsymbol{x}, \tau) \in \kappa_{\tau}(\mathcal{B}), \quad \boldsymbol{x}=\chi(X, t) \in \kappa_{t}(\mathcal{B}) .
$$

It can also be regarded as the relative deformation at time $\tau$ with respect to the present configuration at time $t$ as denoted by $\chi_{t}(\boldsymbol{x}, \tau)$ and its gradient

$$
F_{t}(\boldsymbol{x}, \tau)=\nabla_{x} \chi_{t}(\boldsymbol{x}, \tau)
$$


is called the relative deformation gradient (see [7, 22]). One can easily show that

$$
F_{t}(\boldsymbol{x}, \tau)=\nabla_{X}(\chi(X, \tau)) \nabla_{X}(\chi(X, t))^{-1}=F(X, \tau) F(X, t)^{-1} .
$$

We also define the relative displacement vector $\boldsymbol{\xi}-\boldsymbol{x}$ as

$$
\boldsymbol{u}_{t}(\boldsymbol{x}, \tau)=\chi_{t}(\boldsymbol{x}, \tau)-\boldsymbol{x} .
$$

Note that $\boldsymbol{u}_{t}(\boldsymbol{x}, t)=0$ and

$$
\nabla_{x} \boldsymbol{u}_{t}(\boldsymbol{x}, \tau)=F_{t}(\boldsymbol{x}, \tau)-I=F(X, \tau) F(X, t)^{-1}-I,
$$

hence, we have

$$
F_{t}(\tau)=I+H_{t}(\tau) \quad \text { or } \quad F(\tau)=\left(I+H_{t}(\tau)\right) F(t),
$$

where $I$ is the identity tensor and

$$
H_{t}(\boldsymbol{x}, \tau)=\nabla_{x} \boldsymbol{u}_{t}(\boldsymbol{x}, \tau)
$$

is the relative displacement gradient at time $\tau$ with respect to the present configuration $\kappa_{t}$ (emphasize, not $\left.\kappa_{0}\right)$. Note that $F_{t}(t)=I$ and $H_{t}(t)=0$.

Moreover, by taking the time derivative with respect to $\tau$, it gives

$$
\dot{F}(\tau)=\dot{H}_{t}(\tau) F(t)
$$

In these expressions and hereafter, we shall often denote a function $f$ as $f(t)$ to emphasize its value at time $t$ when its spatial variable is self-evident.

In summary, we can represent the deformation and deformation gradient schematically in the following diagram:

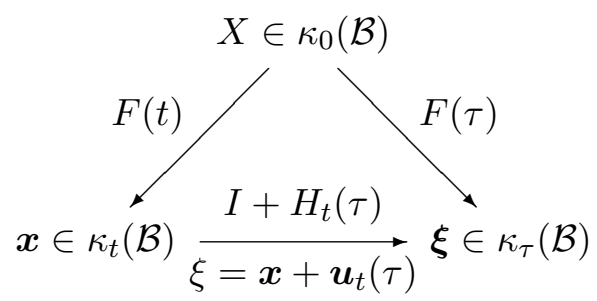

On the other hand, from (12) we have

$$
\chi(X, \tau)=\chi(X, t)+\boldsymbol{u}_{t}(\chi(X, t), \tau)
$$

and hence by taking time derivative with respect to $\tau$, the acceleration at the instant $\tau$ becomes

$$
\ddot{\boldsymbol{x}}(X, \tau)=\ddot{\boldsymbol{u}}_{t}(\boldsymbol{x}, \tau) .
$$


Similarly, for thermal variables, we can define the relative temperature,

$$
\theta_{t}(\tau)=\theta(\tau)-\theta(t)
$$

and the relative temperature gradient,

$$
\boldsymbol{g}_{t}(\tau)=\nabla_{x} \theta_{t}(\tau)=F(t)^{-T}\left(\nabla_{X} \theta(\tau)-\nabla_{X} \theta(t)\right)
$$

which implies that

$$
\boldsymbol{g}(\tau)-\boldsymbol{g}(t)=F(t)^{T} \boldsymbol{g}_{t}(\tau) .
$$

Note that $\theta_{t}(t)=0$ and $\boldsymbol{g}_{t}(t)=0$.

The relative motion description has been employed in [11] for large deformation in elastic bodies. In this paper, a straight generalization to thermoelastic bodies will be formulated.

\section{Relative Lagrangian formulation}

Regarding the present configuration $\kappa_{t}$ as the reference configuration, we shall denote the Piola-Kirchhoff stress and the material heat flux at time $\tau$ by $T_{t}(\boldsymbol{x}, \tau)$ and $\boldsymbol{q}_{t}(\boldsymbol{x}, \tau)$ respectively with respect to the present configuration $\kappa_{t}$, instead of $T_{\kappa_{t}}(\boldsymbol{x}, \tau)$ and $\boldsymbol{q}_{\kappa t}(\boldsymbol{x}, \tau)$ as defined in (11) for simplicity,

$$
T_{t}=\left(\operatorname{det} F_{t}\right) T F_{t}^{-T}, \quad \boldsymbol{q}_{t}=\left(\operatorname{det} F_{t}\right) F_{t}^{-1} \boldsymbol{q} .
$$

Note that $T_{t}(\boldsymbol{x}, t)=T(\boldsymbol{x}, t)$ reduces to the Cauchy stress, and $\boldsymbol{q}_{t}(\boldsymbol{x}, t)=\boldsymbol{q}(\boldsymbol{x}, t)$, in the Eulerian description.

By the use of the relations (12) through (18), the constitutive equations at time $\tau$ relative to the present stat at time $t$ can now be written in the following form: For $\boldsymbol{x} \in \kappa_{t}(B)$ and $\tau>t$,

$$
\begin{aligned}
T_{t}(\boldsymbol{x}, \tau) & =\mathbb{T}\left(\nabla_{x} \boldsymbol{u}_{t}(\boldsymbol{x}, \tau), \theta_{t}(\boldsymbol{x}, \tau) ; \boldsymbol{x}, t\right), \\
\eta(\boldsymbol{x}, \tau) & =\mathbb{H}\left(\nabla_{x} \boldsymbol{u}_{t}(\boldsymbol{x}, \tau), \theta_{t}(\boldsymbol{x}, \tau) ; \boldsymbol{x}, t\right), \\
\boldsymbol{q}_{t}(\boldsymbol{x}, \tau) & =\mathbb{Q}\left(\nabla_{x} \boldsymbol{u}_{t}(\boldsymbol{x}, \tau), \theta_{t}(\boldsymbol{x}, \tau), \nabla_{x} \theta_{t}(\boldsymbol{x}, \tau) ; \boldsymbol{x}, t\right),
\end{aligned}
$$

where the last arguments $(\boldsymbol{x}, t)$ stand for their dependence on the values of $(F, \theta, \boldsymbol{g})$ at the reference present state, which are assumed to be known functions in this formulation.

\subsection{Boundary value problem}

Let $\Omega=\left\{\boldsymbol{x} \in \kappa_{t}(\mathcal{B})\right\} \subset \mathbb{R}^{3}$ be the region occupied by the body at the present configuration $\kappa_{t}$, and $\partial \Omega=\Gamma_{1} \cup \Gamma_{2}=\Gamma_{3} \cup \Gamma_{4}$ be the disjoint unions of its boundary. Let $\boldsymbol{n}(\boldsymbol{x}, t)$ be the exterior unit normal to $\partial \Omega$ at the present time. 
At time $\tau>t$, we shall consider an initial boundary value problem in Lagrangian formulation (10), with the present state at time $t$ as the reference configuration, given by the following mechanical and thermal problems simultaneously:

$$
\begin{aligned}
& \left\{\begin{array}{l}
\rho(t) \ddot{\boldsymbol{u}}_{t}(\tau)-\operatorname{div}_{x} T_{t}(\tau)=\rho(t) \boldsymbol{b}(\tau), \quad \text { in } \Omega, \\
T_{t}(\tau) \boldsymbol{n}(t)=\boldsymbol{f}(\tau), \quad \text { on } \Gamma_{1}, \\
\boldsymbol{u}_{t}(\tau)=\boldsymbol{d}(\tau), \quad \text { on } \Gamma_{2}, \\
\boldsymbol{u}_{t}(t)=0, \quad \dot{\boldsymbol{u}}_{t}(t)=\boldsymbol{v}(t), \quad \text { in } \Omega,
\end{array}\right. \\
& \left\{\begin{array}{l}
\rho(t) \theta(\tau) \dot{\eta}(\tau)+\operatorname{div}_{x} \boldsymbol{q}_{t}(\tau)=\rho(t) r(\tau), \quad \text { in } \Omega, \\
\boldsymbol{q}_{t}(\tau) \cdot \boldsymbol{n}(t)=0, \quad \text { on } \Gamma_{3}, \\
\theta_{t}(\tau)=h(\tau), \quad \text { on } \Gamma_{4}, \\
\theta_{t}(t)=0, \quad \text { in } \Omega .
\end{array}\right.
\end{aligned}
$$

The body is subjected to the surface traction $\boldsymbol{f}(\boldsymbol{x}, \tau)$, the boundary displacement $\boldsymbol{d}(\boldsymbol{x}, \tau)$, the relative surface temperature $h(\boldsymbol{x}, \tau)$ on the respective parts of $\partial \Omega$ at time $\tau>t$, and the initial velocity $\boldsymbol{v}(\boldsymbol{x}, t)$ in $\Omega$ at the present time $t$. Note that unlike the explicit time dependence in the above expressions, the spatial dependence is implicitly understood and does not explicitly indicated for simplicity.

In these relations, for simplicity, $\operatorname{div}_{x}$ stands for the divergence operator relative to the coordinate $\boldsymbol{x} \in \kappa_{t}(B)$, which is the same as the operator $\operatorname{div}_{\kappa_{t}}$ in (10) for the reference configuration $\kappa_{t}$ in this case.

Together with the constitutive equations (20), the mechanical problem (A) and the thermal problem (B) are to be solved for the relative displacement vector $\boldsymbol{u}_{t}(\tau)$ and the relative temperature $\theta_{t}(\tau)$. Since the constitutive functions $\mathbb{T}, \mathbb{H}$, and $\mathbb{Q}$ in (20) are generally nonlinear for finite deformations, the partial differential equations of these problems are genuinely nonlinear. However, in the relative Lagrangian formulation, for small enough incremental time $\Delta t=\tau-t$, we can easily linearize the constitutive equations relative to the present state at time $t$, so that the boundary value problem becomes linear.

\subsection{Linearized constitutive equations}

Instead of the constitutive equations given in (20) which depend on the state of the body at the present time, it is usually expressed relative to a preferred reference configuration, which characterizes the material symmetry. Let $\kappa_{0}$ be the preferred reference configuration of a thermoelastic body $\mathcal{B}$, and let the Cauchy stress $T(X, t)$ be given by the constitutive equation in the configuration $\kappa_{0}$,

$$
T(X, t)=T(F(X, t), \theta(X, t)) .
$$

We shall regard the present configuration $\kappa_{t}$ as the (updated) reference configuration, and consider a small deformation relative to the present state $\kappa_{t}(\mathcal{B})$ at time 
$\tau=t+\Delta t$ for small enough $\Delta t$. In other words, we shall assume that the relative displacement gradient $H_{t}(\tau)$ and the relative temperature $\theta_{t}(\tau)$ are small, $\left|H_{t}(\tau)\right| \ll 1$ and $\left|\theta_{t}(\tau)\right| \ll 1$, so that we can linearize the constitutive equation (23) at time $\tau$ relative to the reference configuration at time $t$,

$$
\begin{aligned}
T(\tau)=T(F(\tau), \theta(\tau)) & \\
=T(F(t), \theta(t)) & +\partial_{F} T(F(t), \theta(t))[F(\tau)-F(t)] \\
& +\partial_{\theta} T(F(t), \theta(t))[\theta(\tau)-\theta(t)]+o(2),
\end{aligned}
$$

or by the use of (13) and (17),

$$
T(\tau)=T(t)+\partial_{F} T(t)\left[H_{t}(\tau) F(t)\right]+\partial_{\theta} T(t) \theta_{t}(\tau)+o(2),
$$

where $o(2)$ represents higher order terms in the small displacement gradient $\left|H_{t}(\tau)\right|$ and the small relative temperature $\left|\theta_{t}(\tau)\right|$.

The linearized constitutive equation can now be written as

$$
T(\tau)=T(t)+C(F(t), \theta(t))\left[H_{t}(\tau)\right]+P(F(t), \theta(t)) \theta_{t}(\tau)
$$

where

$$
C(F, \theta)[H]:=\partial_{F} T(F, \theta)[H F], \quad P(F, \theta):=\partial_{\theta} T(F, \theta),
$$

define the fourth order elasticity tensor $C(F, \theta)$ and the second order thermal stress coefficient tensor $P(F, \theta)$ relative to the present configuration $\kappa_{t}$.

Similarly, for the constitutive equation of the heat flux $\boldsymbol{q}(X, t)$,

$$
\boldsymbol{q}(X, t)=\boldsymbol{q}(F(X, t), \theta(X, t), \boldsymbol{g}(X, t)),
$$

we have, by assuming small relative temperature and relative temperature gradient,

$$
\begin{aligned}
\boldsymbol{q}(\tau)= & \boldsymbol{q}(F(\tau), \theta(\tau), \boldsymbol{g}(\tau)) \\
=\boldsymbol{q}(t) & +\partial_{F} \boldsymbol{q}(t)[F(\tau)-F(t)] \\
& +\partial_{\theta} \boldsymbol{q}(t)(\theta(\tau)-\theta(t))+\partial_{\boldsymbol{g}} \boldsymbol{q}(t)(\boldsymbol{g}(\tau)-\boldsymbol{g}(t))+o(2),
\end{aligned}
$$

and $o(2)$ stands also for second order terms in the relative temperature gradient $\left|\boldsymbol{g}_{t}(\tau)\right|$. Therefore, the linearized heat flux becomes

$$
\boldsymbol{q}(\tau)=\boldsymbol{q}(t)+A(t)\left[H_{t}(\tau)\right]+\boldsymbol{h}(t) \theta_{t}(\tau)-K(t) \boldsymbol{g}_{t}(\tau)
$$

where

$$
\begin{aligned}
\boldsymbol{h}(t) & :=\partial_{\theta} \boldsymbol{q}(t), \\
K(t) & :=-\partial_{\boldsymbol{g}} \boldsymbol{q}(t) F(t)^{T} . \\
A(t)\left[H_{t}(\tau)\right] & :=\partial_{F} \boldsymbol{q}(t)\left[H_{t}(\tau) F(t)\right],
\end{aligned}
$$

Note that $\boldsymbol{h}, K$, and $A$ are first, second, and third order tensor material quantities respectively. The material function $K$ is called the thermal conductivity tensor. 
For the entropy density $\eta(F, \theta)$, we have

$$
\begin{aligned}
\eta(\tau) & =\eta(t)+\partial_{F} \eta(t) \cdot(F(\tau)-F(t))+\partial_{\theta} \eta(t)(\theta(\tau)-\theta(t))+o(2) \\
& =\eta(t)+\partial_{F} \eta(t) \cdot\left(H_{t}(\tau) F(t)\right)+\partial_{\theta} \eta(t) \theta_{t}(\tau)+o(2)
\end{aligned}
$$

From (6), we have

$$
-\frac{\partial \eta}{\partial F}=\frac{\partial}{\partial F}\left(\frac{\partial \psi}{\partial \theta}\right)=\frac{\partial}{\partial \theta}\left(\frac{1}{\rho} T F^{-T}\right)=\frac{1}{\rho} \frac{\partial T}{\partial \theta} F^{-T}, \quad \frac{\partial \eta}{\partial \theta}=\frac{1}{\theta} \frac{\partial \varepsilon}{\partial \theta} .
$$

Hence, by differentiation with respect to $\tau$, we obtain

$$
\dot{\eta}(\tau)=-\frac{1}{\rho(t)} P(t) \cdot \dot{H}_{t}(\tau)+\frac{c(t)}{\theta(t)} \dot{\theta}_{t}(\tau)+o(2)
$$

where the thermal stress coefficient tensor $P(F . \theta)$ is defined in (25) and the specific heat $c(F, \theta)$ is defined as

$$
c(F, \theta):=\partial_{\theta} \varepsilon(F, \theta) .
$$

Note that $\dot{\eta}(\tau)$ is a first order quantity, so that $\theta(\tau) \dot{\eta}(\tau)=\theta(t) \dot{\eta}(\tau)+o(2)$, and hence, we obtain

$$
\rho(t) \theta(\tau) \dot{\eta}(\tau)=-\theta(t) P(t) \cdot \dot{H}_{t}(\tau)+\rho(t) c(t) \dot{\theta}_{t}(\tau)+o(2)
$$

Furthermore, by the use of (13),

$$
F_{t}(\tau)=I+H_{t}(\tau), \quad \operatorname{det} F_{t}(\tau)=1+\operatorname{tr} H_{t}(\tau),
$$

the Piola-Kirchhoff stress and the heat flux defined in (19) relative to the reference present configuration $\kappa_{t}$ at the instant $\tau$,

$$
\begin{aligned}
& T_{t}(\tau)=\operatorname{det} F_{t}(\tau) T(\tau) F_{t}(\tau)^{-T} \\
& \boldsymbol{q}_{t}(\tau)=\operatorname{det} F_{t}(\tau) F_{t}(\tau)^{-1} \boldsymbol{q}(\tau) .
\end{aligned}
$$

can now be written as

$$
\begin{aligned}
& T_{t}(\tau)=T(t)+L(t)\left[H_{t}(\tau)\right]+P(t) \theta_{t}(\tau), \\
& \boldsymbol{q}_{t}(\tau)=\boldsymbol{q}(t)+G(t)\left[H_{t}(\tau)\right]+\boldsymbol{h}(t) \theta_{t}(\tau)-K(t) \boldsymbol{g}_{t}(\tau),
\end{aligned}
$$

from (24) and (27), and where

$$
\begin{aligned}
& L(t)[H]=(\operatorname{tr} H) T(t)-T(t) H^{T}+C(t)[H], \\
& G(t)[H]=(\operatorname{tr} H) \boldsymbol{q}(t)-H \boldsymbol{q}(t)+A(t)[H] .
\end{aligned}
$$

Note that $L(t)$ and $P(t)$ are functions of $(F(t), \theta(t))$, while $K(t), G(t)$ and $\boldsymbol{h}(t)$ depend on $(F(t), \theta(t), \boldsymbol{g}(t))$. All the functions are supposed to be known at the present state at time $t$ being regarded as the reference state. 


\subsection{Linearized partial differential equations}

By regarding the present state as the reference state, it is assumed that the state variables are all known functions at the present time $t$. That include the deformation gradient $F(t)$, the temperature $\theta(t)$ and their derivatives $\dot{F}(t), \dot{\theta}(t)$, as well as the stress $T(t)$ and the heat flux $\boldsymbol{q}(t)$.

By the use of linearization (30) and (31), the partial differential equations of the problems (A) and (B) become

$$
\left\{\begin{aligned}
\rho(t) \ddot{\boldsymbol{u}}_{t}(\tau)-\operatorname{div}_{x}\left(L(t)\left[\nabla_{x} \boldsymbol{u}_{t}(\tau)\right]+P(t) \theta_{t}(\tau)\right)=\operatorname{div}_{x} T(t)+\rho(t) \boldsymbol{b}(\tau) \\
\rho(t) c(t) \dot{\theta}_{t}(\tau)-\operatorname{div}_{x}\left(K(t) \nabla_{x} \theta_{t}(\tau)-G(t)\left[\nabla_{x} \boldsymbol{u}_{t}(\tau)\right]-\boldsymbol{h}(t) \theta_{t}(\tau)\right) \\
-\theta(t) P(t) \cdot \nabla_{x} \dot{\boldsymbol{u}}_{t}(\tau)=-\operatorname{div}_{x} \boldsymbol{q}(t)+\rho(t) r(\tau)
\end{aligned}\right.
$$

where the relevant material functions $(L, P, c, K, G, \boldsymbol{h})$, defined in (25), (28), (29) and (32), depending on the constitutive functions $(T(F, \theta), \boldsymbol{q}(F, \theta, \boldsymbol{g}), \varepsilon(F, \theta))$, are summarized below,

$$
\begin{aligned}
L(t)[H] & =(\operatorname{tr} H) T(t)-T(t) H^{T}+\frac{\partial T}{\partial F}(t)[H F(t)], \\
G(t)[H] & =(\operatorname{tr} H) \boldsymbol{q}(t)-H \boldsymbol{q}(t)+\frac{\partial \boldsymbol{q}}{\partial F}(t)[H F(t)],
\end{aligned}
$$

for any second order tensor $H$, and

$$
\begin{aligned}
P(t) & =\frac{\partial T}{\partial \theta}(t), & K(t) & =-\frac{\partial \boldsymbol{q}}{\partial \boldsymbol{g}}(t) F(t)^{T}, \\
\boldsymbol{h}(t) & =\frac{\partial \boldsymbol{q}}{\partial \theta}(t), & c(t) & =\frac{\partial \varepsilon}{\partial \theta}(t) .
\end{aligned}
$$

The equations (33) form a system of linear partial differential equations for the relative displacement vector $\boldsymbol{u}_{t}(\boldsymbol{x}, \tau)$ and the relative temperature $\theta_{t}(\boldsymbol{x}, \tau)$ to be solved with the corresponding initial boundary conditions in the problems (A) and (B), for which the state variables of the body at time $t$ are known and the external supplies $(\boldsymbol{b}(\tau), r(\tau))$ are given so that the right hand sides of the system (33) are known quantities.

In this linearization, we do not assume the deformation nor the temperature gradient are small, rather only the relative displacement gradient and the relative temperature gradient with respect to the present state are assumed to be small. This is the idea of "small-on-large", the same as the well-known problem of small deformations superposed on finite deformation in the literature [5]. Therefore, the overall deformation or the temperature gradient may be of finite values, in contrast to the usual theory of linear thermoelasticity which linearizes the problem with respect to the fixed reference configuration assuming small deformation gradient and small temperature gradient. 


\subsection{A Mooney-Rivlin thermoelastic material}

The material functions in (34) amd (35) depend on the constitutive functions $T(F, \theta)$ and $\boldsymbol{q}(F, \theta, \boldsymbol{g})$ relative the the preferred configuration $\kappa_{0}$. We shall consider the isotropic thermoelastic material bodies as an example, for which the general constitutive equations are given by

$$
\begin{aligned}
& T(F, \theta)=s_{0} I+s_{1} B+s_{2} B^{-1}, \\
& \boldsymbol{q}(F, \theta, \boldsymbol{g})=-\left(k_{0} I+k_{1} B+k_{2} B^{-1}\right) \mathfrak{g},
\end{aligned}
$$

where $B=F F^{T}$ is the left Cauchy-Green strain tensor and $\mathfrak{g}=\nabla_{x} \theta=F^{-T} \boldsymbol{g}$ is the temperature gradient with respective to the present configuration $\kappa_{t}$. These are the general representation of isotropic functions as consequence of material objectivity and material symmetry, in which, the material coefficients are scalar isotropic functions [7].

$$
\begin{aligned}
s_{i} & =s_{i}\left(\theta, I_{B}, I I_{B}, I I I_{B}\right), \\
k_{i} & =k_{i}\left(\theta, I_{B}, I I_{B}, I I I_{B}, \mathfrak{g} \cdot \mathfrak{g}, \mathfrak{g} \cdot B \mathfrak{g}, \mathfrak{g} \cdot B^{-1} \mathfrak{g}\right),
\end{aligned}
$$

where $\left(I_{B}, I I_{B}, I I I_{B}\right)$ are the three principal invariants of $B$.

To be more specific, we shall assume that $s_{i}$ and $k_{i}$ are functions of $\theta$ only except $s_{0}=s_{0}\left(I I I_{B}, \theta\right)$ or $s_{0}=s_{0}(\rho, \theta)$ since $\rho=\rho_{0} / \sqrt{I I I_{B}}=\rho_{0} /|\operatorname{det} F|$. This material model is motivated by the well-known Mooney-Rivlin elastic material model [12], and will be referred to as a Mooney-Rivlin thermoelastic material model.

By definition, for any function $\mathfrak{F}(F)$, the gradient is given by,

$$
\frac{\partial \mathfrak{F}}{\partial F}[H]=\left.\frac{d}{d \tau} \mathfrak{F}(F+\tau H)\right|_{\tau=0} \quad \forall H,
$$

from which by the use of the results,

$$
\begin{aligned}
& \left.\frac{d}{d \tau} \operatorname{det}(F+\tau H)\right|_{\tau=0}=(\operatorname{det} F) \operatorname{tr}\left(H F^{-1}\right), \\
& \left.\frac{d}{d \tau}(F+\tau H)^{-1}\right|_{\tau=0}=-F^{-1} H F^{-1}
\end{aligned}
$$

it follows that

$$
\begin{aligned}
& \frac{\partial T}{\partial F}[H]=\beta \operatorname{tr}\left(H F^{-1}\right) I+s_{1}\left(H F^{T}+F H^{T}\right)-s_{2}\left(B^{-1} H F^{-1}+F^{-T} H^{T} B^{-1}\right), \\
& \frac{\partial \boldsymbol{q}}{\partial F}[H]=-k_{1}\left(H F^{T}+F H^{T}\right) \mathfrak{g}+k_{2}\left(B^{-1} H F^{-1}+F^{-T} H^{T} B^{-1}\right) \mathfrak{g},
\end{aligned}
$$

where $\beta=-\rho\left(\partial s_{0} / \partial \rho\right)$. On the other hand, with $\mathfrak{g}=F^{-T} \boldsymbol{g}$, we have the gradient,

$$
\frac{\partial \boldsymbol{q}}{\partial \boldsymbol{g}}=-\left(k_{0} I+k_{1} B+k_{2} B^{-1}\right) F^{-T}
$$


Therefore, from (34) and (35) $)_{2}$ we have

$$
\begin{aligned}
L(t)[H]= & (\operatorname{tr} H) T(t)-T(t) H^{T}+\beta \operatorname{tr}(H) I \\
& +s_{1}\left(H B(t)+B(t) H^{T}\right)-s_{2}\left(B(t)^{-1} H+H^{T} B(t)^{-1}\right), \\
G(t)[H]= & (\operatorname{tr} H) \boldsymbol{q}(t)-H \boldsymbol{q}(t) \\
& -k_{1}\left(H B(t)+B(t) H^{T}\right) \mathfrak{g}(t)+k_{2}\left(B(t)^{-1} H+H^{T} B(t)^{-1}\right) \mathfrak{g}(t), \\
K(t)= & k_{0} I+k_{1} B+k_{2} B^{-1} .
\end{aligned}
$$

Other material function $P(t)$ and others, can easily obtained from the rest of (35).

Note that $L(t)$ is the fourth order elasticity tensor relative to the present state, and $K(t)$ is known as the thermal conductivity tensor. These are the essential features, while the thermal expansion tensor $P(t)$ and the third order tensor $G(t)$ represent the thermo-mechanical coupling effects in the governing equations (33) of thermoelasticity.

\section{Remarks on successive linear approximation}

"In large deformation the stress is built up by summation of linear increments from the stresses in the infinitesimally preceding configurations occupied in the deformation process."

$$
\text { - Truesdell, ([22] Sect. 100, P. 407) }
$$

In an attempt to deal with large deformation, the initial boundary value problem can be solved as a sequence of small incremental problems at every incremental step. In such a manner, the boundary value problem of the system (A) and (B) with linearized partial differential equations (33) can be solved successively with the reference configuration updated to the present state at every time step. We called this a Successive Linear Approximation (SLA) for large deformation [11, 13, 14].

The method of SLA has been treated from three different aspects: physical, mathematical and numerical.

- On physical aspect: It is based on the relative Lagrangian formulation and small-on-large idea, updating the reference configuration at every incremental time step.

- On mathematical aspect: In [1, 2, 18], analyses of the relative Lagrangian formulation of elasticity and viscoelasticity regarding existence, uniqueness and regularity have been proved explicitly for the proposed Mooney-Rivlin model. Such analyses provide a sound theoretical basis for the realization of the method of SLA.

- On numerical aspect: The SLA has been implemented in numerical simulations with finite elements. We have shown that the relative Lagrangian formulation 
facilitates the numerical viability for successively solving large deformation as a sequence of linear problems.

For numerical simulations, comparison with exact solutions in finite elasticity are considered to validate the method, such as pure shear in [13], bending a rectangular block into a circular section in [11].

The SLA method was proposed originally for the study of large deformation of salt tectonics. It has been applied successfully to simulations of sediment-salt migration, such as the formation of salt diapirs, multiple salt domes [14] and borehole closing [2], involving very large deformation and creep motions, for which the material model has been extended to viscoelastic solid bodies. Although the problems of sediment-salt migration have been widely known and studied in petroleum industry. Unlike our approach, most results for salt tectonics are modeled by regarding the bodies as viscous fluids [15] instead of solid bodies to avoid the numerical difficulty due to large deformation and nonlinearity.

The SLA method and the viscoelastic model for geomechanics has been incorporated into modern numerical transport code for subsurface environmental simulators that consider continuum representations of flow, transport, and reactions in porous media [19, 23, 24], applicable to most of the subsurface environmental benchmark problems. It was also used in the mechanical modeling of fold-trust belt consisting of surface salt structures in China [4].

The method has been applied to elasto-plastic model for large deformation [17]. For thermo-mechanical problems, the SLA method has been implemented in various simulations to study the thermal influence on salt tectonics [20, 21]. Further simulations with more thorough implementation of coupled or decoupled system (A) and (B) for thermoelastic problems are in progress.

\section{References}

[1] Cipolatti, R.A.; Liu, I-S.; Rincon, M.A.: Mathematical analysis of successive linear approximation for Mooney-Rivlin material model in finite elasticity, Journal of Applied Analysis and Computation 2, 363-379 (2012).

[2] Cipolatti, R.A.; Liu, I-S.; Palermo, L.A.; Rincon, M.A.; Rosa, R.M.S.: A boundary value problem arising from nonlinear viscoelasticity: mathematical analysis and numerical simulations. (submitted).

[3] Coleman, B.D.; Noll, W.: The thermodynamics of elastic materials with heat conduction and viscosity. Arch. Ration. Mech. Anal. 13, 167178 (1963).

[4] Colon, C.; Teixeira, M.G. et al.: Surface salt in Kuqa fold-thrust belt, northwestern China: time-lapse surface deformation and mechanical modelling. Proceedings of Fringe 2015 Workshop, Rome, Vol. 1, 2015. 
[5] Green, A.E.; Rivlin R.S.; Shield, R.T.: General theory of small elastic deformations superposed on finite deformations. Pro. Roy. Soc. London, Ser. A, 211, 128-154, (1952).

[6] Gurtin, M.E.; Fried, E.: Anand, L.; The Mechanics and Thermodynamics of Continua, Cambridge University Press, 2010.

[7] Liu, I-S.: Continuum Mechanics, Springer: Berlin Heidelberg, 2002.

[8] Liu, I-S.: Method of Lagrange multipliers for exploitation of the entropy principle. Arch. Ration. Mech. Anal. 46, 131148 (1972).

[9] Liu, I-S, Entropy flux relation for viscoelastic bodies, Journal of Elasticity, 90, 259-270 (2008).

[10] Liu, I-S.: On Entropy Flux of Transversely Isotropic Elastic Bodies, Journal of Elasticity 96, 97104 (2009).

[11] Liu, I-S.: Successive linear approximation for boundary value problems of nonlinear elasticity in relative-descriptional formulation, Int. J. Eng Sci. 49, 635-645 (2011).

[12] Liu, I-S.: A note on the Mooney-Rivlin material model, Continuum Mech. and Thermody. 24, 583-590 (2011).

[13] Liu, I-S.; Cipolatti, R.A.; Rincon, M.A.: Successive linear approximation for finite elasticity, Computational and Applied Mathematics 29, 465-478, (2010).

[14] Liu, I-S.; Cipolatti, R.A.; Rincon, M.A.; Palermo, L.A.: Successive linear approximation for large deformation-Instability of salt migration, Journal of Elasticity, 114, 19-39 (2014).

[15] Massimi, P.; Quarteroni, A.; Saleri, F.E.; Scrofani, G.: Modeling of Salt Tectonics. Comput. Methods Appl. Mech. Eng. 197, 281-293 (2007).

[16] Müller, I.: On the entropy inequality. Arch. Ration. Mech. Anal. 26, 118141 (1967).

[17] Pereira, G.T.A.; Teixeira, M.G.; Liu, I-S.: Modelagem elasto-plástica com o método de aproximaes lineares incrementais. Anais do XXXV Congress Nacional de Matemática Aplicada e Computacional, Natal. Vol. 1, 2014.

[18] Rosa, R.M.S.; Cipolatti, R.A.; Liu, I-S.; Palermo, L.A.; Rincon, M.A.: On the existence, uniqueness and regularity of solutions of a viscoelastic Stokes problem modelling salt rocks, DOI 10.1007/s00245-017-9411-7, Applied Mathematics and Optimization, 1-54 (2017)

[19] Steefel, C.I.; Yeh, G.T.; et al.: Reactive transport codes for subsurface environmental simulation. DOI 10.1007/s10596-014-9443-x, Computational Geosciences, $19,445-478(2015)$ 
[20] Teixeira, M.G.; Liu, I-S.; Rincon, M.A.; Cipolatti, R.A.; Palermo, L.A.: The Influence of temperature on the formation of salt domes. International Journal of Modeling and Simulation for Petroleum Industry, 8, 35-41 (2014).

[21] Teixeira, M.G.; Liu, I-S.; Rincon, M.A.; Cipolatti, R.A.; Palermo, L.A.: Thermo-mechanical coupling for salt domes formation. Proceedings of the 11th World Congress on Computational Mechanics, Barcelona. Vol. 1, 2014.

[22] Truesdell, C.; Noll, W.: The Non-Linear Field Theories of Mechanics, 3rd edition. Springer: Berlin, 2004.

[23] Yeh, G.T.; Tsai, C.H.; Liu, I-S.: A Geo-Mechanics Model for Finite ViscoElastic Materials (GMECH 1.0): Theoretcal Basis and Numerical Approximation. Graduate Institute of Applied Geology, National Central University, Jhongli, Taoyuan 32001, Taiwan (2013)

[24] Yeh, G.T.; Tsai, C.H.: A Three-Dimensional Model of Coupled Fluid Flow, Thermal Transport, Hydrogeochemical Transport, and Geomechanics through Multiple Phase Systems (HYDROGEOCHEM 7.1): Theoretical Basis and Numerical Approximation. Technical Report, DOI: 10.13140/RG.2.1.3339.1125, Graduate Institute of Applied Geology, National Central University, Jhongli, Taoyuan 32001, Taiwan (2015). 